\title{
Statistical Mechanics of the 2-Dimensional Focusing Nonlinear Schrödinger Equation
}

\author{
David C. Brydges ${ }^{1}$, Gordon Slade ${ }^{2}$ \\ ${ }^{1}$ Department of Mathematics, University of Virginia, Charlottesville, VA 22903-3199, USA. \\ E-mail: db5d@virginia.edu \\ ${ }^{2}$ Department of Mathematics and Statistics, McMaster University, Hamilton, ON, Canada L8S \\ 4K1. E-mail: slade@mcmaster.ca
}

Received: 4 March 1996/Accepted: 6 June 1996

\begin{abstract}
We study a natural construction of an invariant measure for the 2-dimensional periodic focusing nonlinear Schrödinger equation, with the critical cubic nonlinearity. We find that a phase transition occurs as the coupling constant defining the strength of the nonlinearity is increased, but that the natural construction, successful for the 1-dimensional case and for the 2-dimensional defocusing case, cannot produce an invariant measure. Our methods rely on an analysis of a statistical mechanical model closely related to the spherical model of Berlin and Kac.
\end{abstract}

\section{Statement of Results}

1.1. Invariant measures. The periodic nonlinear Schrödinger equation can be written

$$
i u_{t}+\Delta u+\frac{1}{2} p \lambda|u|^{p-2} u=0,
$$

where $u=u(x, t)$ is complex-valued and $x$ lies in the $d$-dimensional unit torus $\mathbb{T}^{d}$. The sign of the coupling constant $\lambda$ is important for the behaviour of solutions to (1.1). In the defocusing case, corresponding to $\lambda<0$, there are global existence results. In the focusing case, corresponding to $\lambda>0$, for the problem on $\mathbb{R}^{d}$, there is a critical power $p_{c}=2+4 / d$ such that for $p<p_{c}$ there are global existence results while for $p>p_{c}$ there are finite-time blow-up results. Both behaviours can occur when $p=p_{c}$, depending on the value of $\lambda$ and the initial value of $\|u\|_{2}$. These results [19] are for initial data in $H_{1}$.

This paper is primarily concerned with the focusing case for $d=2$ and $p=$ $p_{c}=4$, and more precisely, with the construction of invariant measures for (1.1). A natural approach to the construction of an invariant measure proceeds as follows. The $L^{2}$-norm

$$
\|u\|_{2}^{2}=\int_{\mathbb{T}^{d}}|u(x, t)|^{2} d^{d} x
$$

and the Hamiltonian

$$
H(u)=\|\nabla u\|_{2}^{2}-\lambda\|u\|_{p}^{p}
$$


are invariants for (1.1) [18]. For $N^{2}>0$, let $\chi_{N^{2}}(y)=1$ if $-\infty<y \leqq N^{2}$ and $\chi_{N^{2}}(y)=0$ otherwise. Then the formal expression

$$
d v(\phi)=\frac{1}{Z} \chi_{N^{2}}\left(\|\phi\|_{2}^{2}\right) e^{-H(\phi)} e^{-m^{2}\|\phi\|_{2}^{2}} \prod_{x \in \mathbb{T}^{2}} d \phi_{R}(x) d \phi_{I}(x),
$$

where $\phi(x)=\phi_{R}(x)+i \phi_{I}(x), m^{2}>0$ and $Z$ is a normalization constant, is formally invariant, in the sense that the measure of a given set of fields remains fixed as the fields evolve according to the dynamics defined by (1.1). Combining the kinetic part of the Hamiltonian with the ill-defined product of Lebesgue measures, and changing the definition of $Z$, gives

$$
d v(\phi)=\frac{1}{Z} \chi_{N^{2}}\left(\|\phi\|_{2}^{2}\right) e^{\lambda\|\phi\|_{p}^{p}} d \mu_{C}(\phi),
$$

where $d \mu_{C}(\phi)$ is the Gaussian measure on two-component fields with covariance $C=\left(-\Delta+m^{2}\right)^{-1}[10]$. In particular, writing $\bar{\phi}(x)=\phi_{R}(x)-i \phi_{I}(x)$,

$$
\int \bar{\phi}(x) \phi(y) d \mu_{C}(\phi)=C(x-y)
$$

and expectations of $\phi \phi$ and $\bar{\phi} \bar{\phi}$ vanish. A constraint such as $\chi_{N^{2}}\left(\|\phi\|_{2}^{2}\right)$ is required for the focusing case $\lambda>0$ to counteract the instability due to the interaction, but can be omitted in the defocusing case.

The Gaussian measure $d \mu_{C}(\phi)$ is supported on tempered distributions for $d=2$, so to make sense of (1.5) it is necessary first to make sense of the norms $\|\phi\|_{2}$ and $\|\phi\|_{p}$ and then to show that $\chi_{N^{2}}\left(\|\phi\|_{2}^{2}\right) e^{\lambda\|\phi\|_{p}^{p}}$ is integrable. This is the problem of construction of the measure. To prove that the measure is invariant involves the additional difficulty of extending the dynamics determined by (1.1) to the irregular fields in the support of the measure.

The study of invariant measures was initiated by Lebowitz, Rose and Speer $[13,14]$ for the 1-dimensional periodic focusing case. They showed that, for the critical power $p=6$ and for fixed $\lambda, \chi_{N^{2}}\left(\|\phi\|_{2}^{2}\right) e^{\lambda\|\phi\|_{6}^{6}}$ is integrable for sufficiently small $N^{2}$ but not for large $N^{2}$. Equivalently, for fixed $N^{2}$ there is integrability for small $\lambda$ but not for large $\lambda$. This constructs a measure for weak coupling, supported on fields with the regularity properties of Brownian paths. Bourgain [6] proved wellposedness of (1.1) for almost every initial condition drawn from the support of the measure, and proved that the measure is invariant. A number of other authors have considered related issues for various 1-dimensional partial differential equations, for example [4, 15, 20, 21].

For the 2-dimensional defocusing case with the critical power $p=4$ and any $\lambda<0$, the construction of the measure (1.5) (without the unnecessary constraint $\chi_{N^{2}}\left(\|\phi\|_{2}^{2}\right)$ ) was an early achievement of the constructive quantum field theory program $[10,16]$ and corresponds to the $\phi_{2}^{4}$ Euclidean quantum field theory. Sense is made of the $|\phi|^{4}$ term by Wick ordering. The measure is absolutely continuous with respect to the Gaussian measure and hence is supported on tempered distributions. Recently, Bourgain [7, 8] proved that this measure is invariant under the dynamics of a Wick ordered analogue of (1.1), which involved proving well-posedness for almost every initial condition drawn from the support of $d \mu_{C}$. 
This paper is concerned with the corresponding construction for the 2-dimensional focusing case, with the critical power $p=4$. Jaffe [11] has considered the subcritical power $p=3$ and showed that for any $\lambda \in \mathbb{R}, m^{2}>0$ and $N^{2}>0$,

$$
\int \chi_{N^{2}}\left(\int_{\mathbb{T}^{2}}: \phi(x)^{2}: d x\right) e^{\lambda \int_{\mathbb{T}^{2}}: \phi(x)^{3}: d x} d \mu_{C}(\phi)<\infty .
$$

This allows for the construction of a measure which is absolutely continuous with respect to the Gaussian measure. Invariance of this measure for a Wick ordered version of (1.1), with $p=3$, has not been proved.

1.2. Results. Rather than attempting to proceed directly with Wick ordered $|\phi|^{2}$ and $|\phi|^{4}$, we introduce an ultra-violet cutoff $a>0$ which regularizes the Gaussian measure. With the cutoff, the regularized measure is supported on continuous fields and there is no difficulty in defining powers of the field. A related procedure is tacit in the definition of Wick ordering with the difference that the cutoff is removed by a limit under the $\phi$ integral, whereas our limit will be outside the integral. Having the limit outside is a broader construction since the limiting measure need not be absolutely continuous relative to the Gaussian. There are various ways possible for introducing the cutoff, and we choose to do so by replacing the covariance $C$ by

$$
C_{a}(x-y)=\left(-\Delta+a^{2} \Delta^{2}+m^{2}\right)^{-1}(x-y) .
$$

The Gaussian measure $d \mu_{C_{a}}$ with this covariance is supported on fields which are Hölder continuous with any exponent less than $(4-d) / 2$ (a proof can be based on the proof of [10, Theorem A.4.4]). We believe that our conclusions would remain unchanged with other choices of cutoff, such as a lattice cutoff.

The Fourier coefficients of the covariance are given by

$$
\hat{C}_{a}(k)=\int_{\mathbb{T}^{d}} e^{2 \pi i k \cdot x} C_{a}(x) d^{d} x=\frac{1}{k^{2}+a^{2} k^{4}+m^{2}}, \quad k \in \mathbb{Z}^{d},
$$

while in position space,

$$
C_{a}(x)=\sum_{k \in \mathbb{Z}^{d}} \frac{e^{2 \pi i k \cdot x}}{k^{2}+a^{2} k^{4}+m^{2}} .
$$

We introduce the notation

$$
S_{a}=S_{a}\left(N^{2}\right)=\int \chi_{N^{2}}\left(\|\phi\|_{2}^{2}\right) d \mu_{C_{a}}(\phi)
$$

and

$$
\langle\cdot\rangle_{a}=\frac{1}{S_{a}} \int \cdot \chi_{N^{2}}\left(\|\phi\|_{2}^{2}\right) d \mu_{C_{a}}(\phi)
$$

Our goal is to study the limit of the measure with expectation $\left\langle\cdot e^{\lambda\|\phi\|_{4}^{4}}\right\rangle_{a} /\left\langle e^{\lambda\|\phi\|_{4}^{4}}\right\rangle_{a}$, as $a \rightarrow 0$. We fix $N^{2}$ and vary $\lambda$. The absence of Wick ordering will be discussed below.

For strong coupling, the following theorem is similar to the result of [13] for the case of $d=1$ and $p=6$. 
Theorem 1.1. There is a function $K\left(\lambda, N^{2}\right)$ which is positive for fixed $\lambda$ and large $N^{2}$, or for fixed $N^{2}$ and large $\lambda$, such that

$$
\int e^{\lambda\|\phi\|_{4}^{4}} \chi_{N^{2}}\left(\|\phi\|_{2}^{2}\right) d \mu_{C_{a}}(\phi) \geqq e^{K\left(\lambda, N^{2}\right) a^{-2}} .
$$

Moreover, for any $\lambda, N^{2}>0, \lim _{\lambda \rightarrow \infty} K\left(\lambda, N^{2}\right)=\lim _{N^{2} \rightarrow \infty} K\left(\lambda, N^{2}\right)=\infty$.

The proof of Theorem 1.1 shows that the divergent behaviour arises from a small set of configurations with very large (negative) energies, as in the corresponding 1-dimensional result of [13]. In fact, it suggests that in the limit the measure concentrates on configurations where $|\phi(x)|^{2}$ becomes a delta function of mass $N^{2}$, centred at a point chosen from the torus with the uniform distribution. A configuration whose limit is two or more delta functions sharing the mass can also contribute $\exp \left[K^{\prime}\left(\lambda, N^{2}\right) a^{-2}\right]$, but $K^{\prime}\left(\lambda, N^{2}\right)<K\left(\lambda, N^{2}\right)$.

To state the result for weak coupling, we first introduce notation. Let $x_{1}, \ldots$, $x_{J} \in \mathbb{T}^{2}$ be distinct. Let

$$
F(\phi)=\prod_{j=1}^{J} \bar{\phi}\left(x_{j}\right)^{m_{J}} \phi\left(x_{j}\right)^{n_{j}}
$$

and define $\langle\cdot\rangle_{0}$ by

$$
\langle F(\phi)\rangle_{0}=\prod_{j=1}^{J}\left\langle\bar{\phi}\left(x_{j}\right)^{m_{j}} \phi\left(x_{j}\right)^{n_{\jmath}}\right\rangle_{0}
$$

where

$$
\left\langle\bar{\phi}(x)^{m} \phi(x)^{n}\right\rangle_{0}= \begin{cases}m ! N^{2 m} & \text { if } m=n \\ 0 & \text { otherwise }\end{cases}
$$

The "expectation" $\langle\cdot\rangle_{0}$ corresponds to an uncountable product of distributions of independent Gaussian random variables, each with covariance $N^{2}$. Define

$$
\lambda_{0}=\lambda_{0}\left(N^{2}\right)=\sup \left\{\lambda \geqq 0: \sup _{0<a<1}\left\langle e^{\lambda\|\phi\|_{4}^{4}}\right\rangle_{a}<\infty\right\} .
$$

Theorem 1.2. $\lambda_{0}>0$, and for complex $\lambda$ with $0<|\lambda|<\lambda_{0}$,

$$
\lim _{a \rightarrow 0}\left\langle F(\phi) e^{\lambda\|\phi\|_{4}^{4}}\right\rangle_{a}=e^{2 \lambda N^{4}}\langle F(\phi)\rangle_{0}
$$

In particular,

$$
\lim _{a \rightarrow 0} \frac{\left\langle F(\phi) e^{\lambda\|\phi\|_{4}^{4}}\right\rangle_{a}}{\left\langle e^{\lambda\|\phi\|_{4}^{4}}\right\rangle_{a}}=\langle F(\phi)\rangle_{0}
$$

is independent of $\lambda$.

The remainder of this paper is organised as follows. In Sect. 1.3 we interpret and discuss Theorems 1.1 and 1.2. Sect. 1.3.2 contains the statement of Lemma 1.3, a result on the "spherical model" with partition function (1.11) which is a key ingredient in the proofs of Theorems 1.1 and 1.2. In Sects. 2 and 3 we reduce the proofs of these two theorems to Lemma 1.3. Finally, in Sect. 4, we analyse the spherical model and prove Lemma 1.3. 


\subsection{Discussion of results}

1.3.1. Role of Wick ordering. Wick ordering with respect to the covariance $C_{a}$ is defined by

$$
\begin{aligned}
& :|\phi(x)|^{2}:=|\phi(x)|^{2}-C_{a}(0), \\
& :|\phi(x)|^{4}:=|\phi(x)|^{4}-4 C_{a}(0)|\phi(x)|^{2}+2 C_{a}(0)^{2} .
\end{aligned}
$$

Wick ordering in the constraint replaces $\chi_{N^{2}}\left(\|\phi\|_{2}^{2}\right)$ by $\chi_{N^{2}+C_{a}(0)}\left(\|\phi\|_{2}^{2}\right)$. As $a \rightarrow 0, C_{a}(0)$ diverges logarithmically. Hence Wick ordering in the constraint corresponds to an asymptotic regime of Theorem 1.1. This conclusion is not changed by Wick ordering the interaction in Theorem 1.1, because $4 C_{a}(0)\|\phi\|_{2}^{2} \leqq 4 C_{a}(0)\left(N^{2}+\right.$ $\left.C_{a}(0)\right)=O\left(|\log a|^{2}\right)$, which cannot overcome the $O\left(a^{-2}\right)$ in Theorem 1.1. The divergent lower bound of Theorem 1.1 implies that no limit in this regime can be absolutely continuous relative to the Gaussian measure $d \mu_{C_{a}}$ at $a=0$, with or without Wick ordering in the constraint and/or interaction. Further comments appeared below Theorem 1.1.

Theorem 1.2 closes off the possibility of limits as $a \rightarrow 0$ with $\lambda$ fixed and small and no Wick ordering in the constraint. This expectation cannot be associated with a measure on any acceptable space of initial data, because all points are independent, so the objects it lives on cannot even be distributions. Wick ordering the interaction will not help: the constant term $2 C_{a}(0)^{2}$ cancels in numerator and denominator and the $4 C_{a}(0)|\phi(x)|^{2}$ is the same as taking $m^{2}=O(|\log a|)$. In our proof of this theorem $m^{2}$ enters in an essential way only in the combination $a^{2} m^{2}$, and the theorem's conclusion is unchanged with a logarithmically divergent mass.

There remains the possibility, which we do not address, that there are limits in which $N^{2} \rightarrow \infty$ and $\lambda \rightarrow 0$ as $a \rightarrow 0$, in such a way as to correspond to the critical point separating the weak and strong coupling regimes. Another possibility is that there are invariant measures akin to the microcanonical distribution in which the Hamiltonian is constrained as well as $\|\phi\|_{2}^{2}$.

1.3.2. The spherical model. Theorem 1.2 shows that the behaviour for small $\lambda$ is the same as the behaviour for $\lambda=0$, i.e., the interaction plays no role in the limit. For $\lambda=0$, the left side of (1.17) reduces to

$$
\frac{1}{S_{a}} \int F(\phi) \chi_{N^{2}}\left(\|\phi\|_{2}^{2}\right) d \mu_{C_{a}}(\phi) .
$$

For the case of $d=2$, this expectation is a version of the spherical model of Berlin and $\mathrm{Kac}[3,2]$.

The Berlin-Kac spherical model in $d$ dimensions is a model of spins $\varphi_{x} \in \mathbb{R}$ un a $d$-dimensional lattice with unit spacing and $a^{-d}$ sites. The partition function is the expectation with respect to a Gaussian measure of the constraint $\delta\left(\sum_{x} \varphi_{x}^{2}-a^{-d}\right)$. This corresponds in our continuum language to taking $a=1$ in the covariance $C_{a}$, working on a torus of volume $a^{-d}$, and using the constraint $\delta\left(\|\phi\|_{2}^{2}-N^{2} a^{-d}\right)$. Scaling this partition function to restore the volume to 1 , followed by scaling the field, also restores the factor $a^{2}$ to the covariance and yields our partition function with $N^{2}$ replaced by $N^{2} a^{2-d}$. Thus our partition function corresponds to that of the Berlin-Kac model if and only if $d=2$.

Berlin and Kac evaluated the free energy of their spherical model exactly. We will analyse the spherical model (1.21) in detail in Sect. 4. Using contour 
integration, the asymptotic behaviour of the partition function $S_{a}$ will be determined, as well as that of the correlation functions (1.21), as in the following lemma whose proof is given in Sect. 4.

Lemma 1.3. (a) For any $N^{2}>0$ there is an explicitly computable function $\alpha\left(N^{2}\right)>0$ such that

$$
\lim _{a \rightarrow 0} a^{2} \log S_{a}\left(N^{2}\right)=-\alpha\left(N^{2}\right) .
$$

Corrections to this law are also computable.

(b) If $\lambda<N^{-4}$,

$$
\lim _{a \rightarrow 0}\left\langle|\phi(0)|^{2} e^{\lambda N^{2}|\phi(0)|^{2}}\right\rangle_{a}=\frac{N^{2}}{\left(1-\lambda N^{4}\right)^{2}} .
$$

(c) For $F(\phi)$ of the form (1.13),

$$
\lim _{a \rightarrow 0}\langle F(\phi)\rangle_{a}=\langle F(\phi)\rangle_{0}
$$

The failure to produce a limiting measure for small $\lambda$ can be understood to arise from an incompatibility of the constraint and the limit $a \rightarrow 0$ of the Gaussian measure. For $\lambda=0$, this incompatibility finds expression in (1.22), which states that the probability with respect to the Gaussian measure $d \mu_{C_{a}}$ that the field obeys the constraint decays exponentially fast in $a^{-2}$. In the limit, the Gaussian measure is supported on tempered distributions which cannot obey the constraint.

For $\lambda=0$, this incompatibility can also be understood from the perspective of statistical mechanics, as follows. For $d=2$, there is no phase transition for the spherical model and the critical temperature is $T_{c}=0[3,2]$. The limit $a \rightarrow 0$ is analogous to a continuum limit for the spherical model, and the breakdown of the limiting "measure" can be understood from the fact that we are essentially taking a continuum limit without simultaneously scaling the temperature to the critical temperature. The correlation length therefore approaches zero in the continuum limit, corresponding to the complete decoupling expressed in Theorem 1.2. In order to get a nontrivial continuum limit, we should drive the temperature to the critical temperature $T_{c}=0$ as we take $a \rightarrow 0$. This is discussed in more detail in Remark 4.5 below, where we argue heuristically that the correct way to do this corresponds to Wick ordering the constraint. However, in view of Theorem 1.1 and the discussion of Sect. 1.3.1, this option will not succeed in producing an invariant measure when $\lambda>0$ is held fixed as $a \rightarrow 0$.

1.3.3. Location of the critical point. For small $\lambda$, according to Theorem 1.2 , the interaction term plays no role in the limit $a \rightarrow 0$. On the other hand, for large $\lambda$, Theorem 1.1 shows that the interaction term plays an overwhelming role. Our methods are insufficient to locate a critical value of $\lambda$ separating these two behaviours. For $d=1$ and $p=6$, Lebowitz, Rose and Speer [13] prove that the corresponding critical point can be expressed in terms of the best constant in the Sobolev inequality (writing $\|\cdot\|$ for norms in $\mathbb{R}^{1}$ )

$$
\|\phi\|_{6}^{6} \leqq C_{1}\|\nabla \phi\|_{2}^{2}\|\phi\|_{2}^{4}
$$


by

$$
\lambda_{c}=\frac{1}{C_{1} N^{4}}
$$

This value of $\lambda_{c}$ is the onset of finite-time blow-up for (1.1) on $\mathbb{R}$ [19]. For $d=1$, $p=6$ and $\lambda<\lambda_{c},(1.1)$ is globally well-posed and the analogue [13, Theorem 2.2] of Theorem 1.2 produces an invariant measure.

For $d=2$, the Sobolev inequality is

$$
\|\phi\|_{4}^{4} \leqq C_{2}\|\nabla \phi\|_{2}^{2}\|\phi\|_{2}^{2}
$$

(norms are on $\mathbb{R}^{2}$ ) and the analogue of $(1.26)$ is

$$
\lambda_{c}=\frac{1}{C_{2} N^{2}}
$$

The constant $C_{2}$ is given in terms of a radial function $\psi$ on $\mathbb{R}^{2}$, which is the ground state of a nonlinear partial differential equation [19], by

$$
\|\psi\|_{4}^{4}=C_{2}\|\nabla \psi\|_{2}^{2}\|\psi\|_{2}^{2} .
$$

We conjecture that the conclusions of Theorems 1.1 and 1.2 hold respectively for $\lambda$ above and below the value $\lambda_{c}$ given in (1.28). This is the critical value for global well-posedness for the nonlinear Schrödinger equation on $\mathbb{R}^{2}$ [19].

1.3.4. Collapse of self-attracting walks. Our results for the nonlinear Schrödinger equation are related to a collapse transition for self-attracting walks studied recently in $[5,9]$ and to the Edwards model with the focusing sign [12].

For an $n$-component field $\phi$, the $|\phi|^{4}$ measure corresponds to the Edwards model of polymers in the formal limit $n \rightarrow 0$ [1]. Le Gall [12] has shown that the Edwards model partition function, with the focusing sign in the interaction, is finite for sufficiently small coupling and infinite for sufficiently large coupling. Bounds are given on the critical point for this transition, but they are not sharp.

Detailed studies have been made of the corresponding transition for a discrete version of the Edwards model with the focusing sign: self-attracting walks. In [9], the case of weak coupling is considered. Theorem 1.2 resembles the self-attracting walk results, but for dimension $d>2$, where the limiting partition function can be evaluated exactly and is essentially trivial. For the self-attracting walk model in $d=2$, the weak coupling regime is not independent of $\lambda$. The strong coupling regime is analysed in [5]. The strong coupling, or collapsed, phase is known to persist to a critical value of the coupling constant determined by the best constant in a Sobolev inequality, as in (1.28). The weak coupling results have not been proved to extend to this same value, but this is conjectured to be the case. This provides circumstantial evidence in favour of the conjecture that the critical point for the nonlinear Schrödinger equation is also determined by the Sobolev constant.

\section{Strong Coupling}

In this section we prove Theorem 1.1, using the method of [13, Theorem 2.2(b)] together with Lemma 1.3(a). The proof involves showing that there is a set of 
fields, with energies of order $-O\left(a^{-2}\right)$, having small but sufficient measure that their contribution alone gives the lower bound of Theorem 1.1.

Proof of Theorem 1.1. Let $\varphi$ be a smooth field on the torus and $\zeta=\phi-\varphi$. Translation in the Gaussian measure gives

$$
d \mu_{C_{a}}(\phi)=\exp \left[-\left(\varphi, C_{a}^{-1} \varphi\right)-2\left(C_{a}^{-1} \varphi, \zeta\right)\right] d \mu_{C_{a}}(\zeta)
$$

Let

$$
E_{a}(\varphi)=\left(\varphi, C_{a}^{-1} \varphi\right)-\lambda\|\varphi\|_{4}^{4}=\|\nabla \varphi\|_{2}^{2}+a^{2}\|\Delta \varphi\|_{2}^{2}+m^{2}\|\varphi\|_{2}^{2}-\lambda\|\varphi\|_{4}^{4} .
$$

By (2.1), there is a function $W(\varphi, \zeta)$, odd in $\zeta$, such that

$$
\begin{aligned}
& \int e^{\lambda\|\phi\|_{4}^{4}} \chi_{N^{2}}\left(\|\phi\|_{2}^{2}\right) d \mu_{C_{a}}(\phi) \\
& =e^{-E_{a}(\varphi)} \int \chi_{N^{2}}\left(\|\varphi+\zeta\|_{2}^{2}\right) e^{W(\varphi, \zeta)+\lambda\|\zeta\|_{4}^{4}+2 \lambda\|\varphi \zeta\|_{2}^{2}+4 \lambda\|\operatorname{Re} \bar{\varphi} \zeta\|_{2}^{2}} d \mu_{C_{a}}(\zeta) \\
& \quad \geqq e^{-E_{a}(\varphi)} \int \chi_{N^{2}}\left(\|\varphi+\zeta\|_{2}^{2}\right) e^{W(\varphi, \zeta)} d \mu_{C_{a}}(\zeta) .
\end{aligned}
$$

To make an appropriate choice for $\varphi$, let $\psi$ be a function on $\mathbb{R}^{2}$ obeying (1.29). Since (1.29) is homogeneous in $\psi$, we may assume $0<\|\psi\|_{2}<N$. The function $\psi$ is positive, radial, belongs to $C^{\infty} \cap H^{1}$, and is a constant multiple of the ground state of the equation $\Delta \psi-\psi+\psi^{3}=0$ [19]. Also, being a radial $H^{1}\left(\mathbb{R}^{2}\right)$ function, $\psi$ obeys the estimate $|\psi(x)| \leqq c|x|^{-1 / 2}\|\psi\|_{H^{1}}(|x| \geqq 1)[17]$, which together with the differential equation implies $\|\Delta \psi\|_{2}<\infty$ (recall that $\|\cdot\|$ denotes norms on $\mathbb{R}^{2}$ ). We regard opposite boundaries of $\left[-\frac{1}{2}, \frac{1}{2}\right]^{2}$ as identified, yielding the torus $\mathbb{T}^{2}$, and choose a smooth function $\eta$ with support in $\left(-\frac{1}{2}, \frac{1}{2}\right)^{2}$ which equals 1 in $\left[-\frac{1}{4}, \frac{1}{4}\right]^{2}$. Let

$$
\varphi_{a}(x)=a^{-1} \psi\left(a^{-1} x\right) \eta(x), \quad x \in \mathbb{T}^{2} .
$$

Then $\varphi_{a}$ is a smooth function on the torus, and

$$
\left\|\varphi_{a}\right\|_{2} \leqq\|\psi\|_{2}<N
$$

Also, as $a \rightarrow 0$,

$$
\begin{aligned}
\left\|\varphi_{a}\right\|_{2}^{2} & =\|\psi\|_{2}^{2}-o(1), \\
\left\|\varphi_{a}\right\|_{4}^{4} & =a^{-2}\left(\|\psi\|_{4}^{4}-o(1)\right), \\
\left\|\nabla \varphi_{a}\right\|_{2}^{2} & =a^{-2}\left(\|\nabla \psi\|_{2}^{2}-o(1)\right), \\
\left\|\Delta \varphi_{a}\right\|_{2}^{2} & =a^{-4}\left(\|\Delta \psi\|_{2}^{2}-o(1)\right) .
\end{aligned}
$$

Using (1.29), this gives

$$
E_{a}\left(\varphi_{a}\right)=a^{-2}\left[\left(1-\lambda C_{2}\|\psi\|_{2}^{2}\right)\|\nabla \psi\|_{2}^{2}+\|\Delta \psi\|_{2}^{2}+o(1)\right] .
$$


Let $\delta=N-\|\psi\|_{2}>0$. Then $\|\zeta\|_{2} \leqq \delta$ implies $\left\|\varphi_{a}+\zeta\right\|_{2} \leqq N$ and hence

$$
\chi_{N^{2}}\left(\left\|\varphi_{a}+\zeta\right\|_{2}^{2}\right) \geqq \chi_{\delta^{2}}\left(\|\zeta\|_{2}^{2}\right) .
$$

Using this inequality to obtain a lower bound on the right side of (2.3), and then applying Jensen's inequality together with the fact that $W$ is odd in $\zeta$, gives the lower bound

$$
\int e^{\lambda\|\phi\|_{4}^{4}} \chi_{N^{2}}\left(\|\phi\|_{2}^{2}\right) d \mu_{C_{a}}(\phi) \geqq e^{-E_{a}\left(\varphi_{a}\right)} S_{a}\left(\delta^{2}\right),
$$

where $S_{a}$ is the spherical model partition function (1.11). By (1.22), $\log S_{a}\left(\delta^{2}\right) \sim$ $-a^{-2} \alpha\left(\delta^{2}\right)$ with $\alpha\left(\delta^{2}\right)>0$. The right side of (2.12) therefore gives an exponentially divergent lower bound of the form $\exp \left[K\left(\lambda, N^{2}\right) a^{-2}\right]$, provided $\lambda$ is sufficiently large that

$$
K\left(\lambda, N^{2}\right)=\left(\lambda C_{2}\|\psi\|_{2}^{2}-1\right)\|\nabla \psi\|_{2}^{2}-\|\Delta \psi\|_{2}^{2}-\alpha\left(\delta^{2}\right)>0 .
$$

For fixed $N^{2}$, clearly $\lim _{\lambda \rightarrow \infty} K\left(\lambda, N^{2}\right)=\infty$. Also, for fixed $\lambda, \lim _{N^{2} \rightarrow \infty} K\left(\lambda, N^{2}\right)$ $=\infty$ (we can take $\|\psi\|_{2}=N-1$ and hence $\delta=1$ for large $\left.N^{2}\right)$.

\section{Weak Coupling}

We will prove Theorem 1.2 using Lemma 1.3 and the following version of the Vitali convergence theorem [16, Proposition VIII.19].

Lemma 3.1. Suppose that $f_{a}(\lambda)$ is a family of functions which are analytic and uniformly bounded in $|\lambda|<\lambda_{0}$. Suppose that $g(\lambda)$ is analytic on the same disk and that $\lim _{a \rightarrow 0} f_{a}^{(n)}(0)=g^{(n)}(0)$ for all $n=1,2,3, \ldots$ Then $f_{a}$ converges to $g(\lambda)$ on the disk.

Proof of Theorem 1.2. To show that $\lambda_{0}$, defined in (1.16), is positive, it suffices to show that there is a positive $\lambda$ and $M$ such that for all small $a$,

$$
\left\langle e^{\lambda\|\phi\|_{4}^{4}}\right\rangle_{a} \leqq M
$$

For this we apply Jensen's inequality in the form

$$
e^{\lambda \int|\phi(x)|^{4} d x} \leqq \int \frac{|\phi(x)|^{2}}{\int|\phi(u)|^{2} d u} e^{\lambda|\phi(x)|^{2} \int|\phi(u)|^{2} d u} d x .
$$

(A similar application of Jensen's inequality was used in [9, Theorem 2.1].) Let $b, \lambda>0$ and $0<B \leqq N^{2}$. Then

$$
\frac{b}{B} e^{\lambda b B}=\frac{b}{B}+\frac{b}{B}\left(e^{\lambda b B}-1\right) \leqq \frac{b}{B}+\frac{b}{N^{2}}\left(e^{\lambda b N^{2}}-1\right) \leqq \frac{b}{B}+\frac{b}{N^{2}} e^{\lambda b N^{2}} .
$$

Choose $b=|\phi(x)|^{2}$ and $B=\|\phi\|_{2}^{2}$, so that (3.2),(3.3) and the constraint imply

$$
e^{\lambda \int|\phi(x)|^{4} d x} \leqq 1+\frac{1}{N^{2}} \int d x|\phi(x)|^{2} e^{\lambda N^{2}|\phi(x)|^{2}} .
$$

Using translation invariance, this gives the upper bound

$$
\left\langle e^{\lambda \int|\phi|^{4}}\right\rangle_{a} \leqq 1+\frac{1}{N^{2}}\left\langle|\phi(0)|^{2} e^{\lambda N^{2}|\phi(0)|^{2}}\right\rangle_{a}
$$


By (1.23) of Lemma 1.3, if $\lambda<N^{-4}$,

$$
\lim _{a \rightarrow 0}\left\langle|\phi(0)|^{2} e^{\lambda N^{2}|\phi(0)|^{2}}\right\rangle_{a}=\frac{N^{2}}{\left(1-\lambda N^{4}\right)^{2}},
$$

which completes the proof that $\lambda_{0}>0$.

To prove (1.17), we apply Lemma 3.1 with $f_{a}(\lambda)=\left\langle F(\phi) e^{\lambda \int|\phi|^{4}}\right\rangle_{a}$ and $g(\lambda)=$ $e^{2 \lambda N^{4}}\langle F(\phi)\rangle_{0}$. For the hypothesis of uniform boundedness, it suffices to show that there exists an $M_{1}$ (depending on $F$ ) such that for $0<\lambda<\lambda_{0}$,

$$
\left\langle|F(\phi)| e^{\lambda\|\phi\|_{4}^{4}}\right\rangle_{a} \leqq M_{1}
$$

This also guarantees the analyticity of the expectations $f_{a}(\lambda)$. By Hölder's inequality, it suffices to obtain an upper bound for each of

$$
\left\langle|F(\phi)|^{S}\right\rangle_{a}, \quad\left\langle e^{\lambda r\|\phi\|_{4}^{4}}\right\rangle_{a},
$$

where $r^{-1}+s^{-1}=1, s$ is an even integer, and $r$ is as close as desired to 1 . The second quantity is bounded for $r \lambda<\lambda_{0}$. For the first quantity in (3.8), it suffices to show that for any $F$ of the form (1.13),

$$
\lim _{a \rightarrow 0}\langle F(\phi)\rangle_{a}=\langle F(\phi)\rangle_{0}
$$

But this is (1.24) of Lemma 1.3.

For the hypothesis in Lemma 3.1 concerning derivatives, it suffices to show that for every $n \geqq 0$,

$$
\left.\lim _{a \rightarrow 0} \frac{d^{n}}{d \lambda^{n}}\right|_{\lambda=0}\left\langle F(\phi) e^{\lambda\|\phi\|_{4}^{4}}\right\rangle_{a}=\left(2 N^{4}\right)^{n}\langle F(\phi)\rangle_{0} .
$$

By the Cauchy integral formula for derivatives we may interchange derivatives with the expectation, so that (3.10) will follow if

$$
\lim _{a \rightarrow 0}\left\langle F(\phi) \prod_{i=1}^{n}\left|\phi\left(y_{i}\right)\right|^{4}\right\rangle_{a}=\left\langle F(\phi) \prod_{i=1}^{n}\left|\phi\left(y_{i}\right)\right|^{4}\right\rangle_{0}
$$

(where the $y_{i}$ 's are distinct, and distinct from the $x_{j}$ 's occurring in $F$ ). The factor $\prod_{i=1}^{n}\left|\phi\left(y_{i}\right)\right|^{4}$ can be absorbed into $F$, which reduces (3.11) to (3.9).

\section{The Spherical Model}

In this section we prove Lemma 1.3. This involves computing the asymptotic behaviour, as $a \rightarrow 0$, of integrals of the form

$$
\int g(\phi) \chi_{N^{2}}\left(\|\phi\|_{2}^{2}\right) d \mu_{C_{a}}(\phi) .
$$

We begin in Sect. 4.1 by discussing a contour integral representation for (4.1). Then in Sect. 4.2 we prove some preliminary lemmas relevant for the contour integral. 
Finally, in Sect. 4.3 we obtain the asymptotic form of the contour integral and give the proof of Lemma 1.3.

4.1. Contour integral representation. For the constraint in (4.1), we use the integral representation

$$
\chi_{N^{2}}(t)=\lim _{A \rightarrow \infty} \frac{1}{2 \pi i} \int_{\Gamma_{\sigma}} d s\left(\frac{1}{s}-\frac{1}{s+A}\right) e^{s\left(N^{2}-t\right)}
$$

for the Heaviside function, where, writing $s=\sigma+i \tau, \Gamma_{\sigma}$ is a contour $\operatorname{Re} s=\sigma$ with $\sigma>0$, oriented in the direction of increasing $\tau$. The integral under the limit is absolutely convergent and evaluates to give a nonnegative function of $t$ that increases monotonically to the Heaviside function.

We assume that $g$ is such that $\int|g(\phi)| e^{-\sigma\|\phi\|_{2}^{2}} d \mu_{C_{a}}(\phi)<\infty$, which implies that (4.1) is finite. By the dominated convergence theorem,

$$
\begin{aligned}
& \int g(\phi) \chi_{N^{2}}\left(\|\phi\|_{2}^{2}\right) d \mu_{C_{a}}(\phi) \\
& \quad=\lim _{A \rightarrow \infty} \int d \mu_{C_{a}}(\phi) g(\phi) \frac{1}{2 \pi i} \int_{\Gamma_{\sigma}} d s\left(\frac{1}{s}-\frac{1}{s+A}\right) e^{s\left(N^{2}-\|\phi\|_{2}^{2}\right)} .
\end{aligned}
$$

By Fubini's theorem, (4.3) is equal to

$$
\lim _{A \rightarrow \infty} \frac{1}{2 \pi i} \int_{\Gamma_{\sigma}} d s\left(\frac{1}{s}-\frac{1}{s+A}\right) e^{s N^{2}} \int d \mu_{C_{a}}(\phi) g(\phi) e^{-s\|\phi\|_{2}^{2}} .
$$

Define

$$
Q(s)=\int d \mu_{C_{a}}(\phi) e^{-s\|\phi\|_{2}^{2}},
$$

which is analytic for $s$ in the right half plane. Let

$$
\hat{C}_{a}^{s}(k)=\frac{1}{k^{2}+a^{2} k^{4}+m^{2}+s}, \quad k \in \mathbb{Z}^{2},
$$

and

$$
\langle g\rangle^{(s)}=\int g(\phi) d \mu_{C_{a}^{s}}(\phi)=\frac{1}{Q(s)} \int g(\phi) e^{-s\|\phi\|_{2}^{2}} d \mu_{C_{a}}(\phi) .
$$

By the above assumption on $g,\langle g\rangle^{(s)}$ exists for $s \in \Gamma_{\sigma}$ and is analytic for $\operatorname{Re} s>\sigma$. Then, scaling $s \rightarrow a^{-2} s$ and restoring the contour, (4.4) equals

$$
\lim _{A \rightarrow \infty} \frac{1}{2 \pi i} \int_{\Gamma_{\sigma}} d s\left(\frac{1}{s}-\frac{1}{s+a^{2} A}\right) e^{a^{-2} s N^{2}} Q\left(a^{-2} s\right)\langle g\rangle^{\left(a^{-2} s\right)} .
$$

The function $Q(s)$ obeys $\frac{d}{d s} \log Q(s)=-C_{a}^{s}(0)$, and hence

$$
\log Q(s)=-\int_{0}^{s} C_{a}^{t}(0) d t
$$

Let

$$
h(a, s)=s N^{2}+a^{2} \log Q\left(a^{-2} s\right)=s N^{2}-\int_{0}^{s} C_{a}^{a^{-2} t}(0) d t .
$$


Then (4.8) is equal to

$$
\lim _{A \rightarrow \infty} \frac{1}{2 \pi i} \int_{\Gamma_{\sigma}} d s\left(\frac{1}{s}-\frac{1}{s+a^{2} A}\right) e^{a^{-2} h(a, s)}\langle g\rangle^{\left(a^{-2} s\right)} .
$$

Define

$$
h(s)=s N^{2}-\int_{0}^{s} d t \int_{\mathbb{R}^{2}} \frac{d^{2} k}{k^{2}+k^{4}+t} .
$$

Then $h(s)=\lim _{a \rightarrow 0} h(a, s)$, since

$$
\begin{aligned}
& \int_{0}^{s} C_{a}^{a^{-2} t}(0) d t=\int_{0}^{s} d t \sum_{k \in \mathbb{Z}^{2}} \frac{1}{k^{2}+a^{2} k^{4}+m^{2}+a^{-2} t} \\
& \quad=\int_{0}^{s} d t \sum_{k \in a \mathbb{Z}^{2}} \frac{a^{2}}{k^{2}+k^{4}+a^{2} m^{2}+t} \rightarrow \int_{0}^{s} d t \int_{\mathbb{R}^{2}} \frac{d^{2} k}{k^{2}+k^{4}+t} \quad \text { as } a \rightarrow 0 .
\end{aligned}
$$

Let

$$
\rho(a, s)=a^{-2}(h(a, s)-h(s)) .
$$

Then by (4.11),

$$
\int g(\phi) \chi_{N^{2}}\left(\|\phi\|_{2}^{2}\right) d \mu_{C_{a}}(\phi)=\lim _{A \rightarrow \infty} \frac{1}{2 \pi i} \int d s\left(\frac{1}{s}-\frac{1}{s+a^{2} A}\right) e^{a^{-2} h(s)+\rho(a, s)}\langle g\rangle^{\left(a^{-2} s\right)} .
$$

We will analyse the right side of (4.15) using the method of steepest descents, as in the original Berlin-Kac study of the spherical model [3]. In preparation for this, in the next section we prove three lemmas.

4.2. Preliminaries. The first lemma provides an analysis of the function $h(s)$. In particular, it shows that $h$ has a critical point $s_{c}>0$; later we will take $\sigma=s_{c}$ in the contour of (4.15).

Lemma 4.1. The function $h(s)$ is analytic in the right half plane, with $h^{\prime}(s)=0$ at a unique critical point $s_{c}>0$. At the critical point, $h\left(s_{c}\right)<0$. Also, $h^{\prime \prime}\left(s_{c}\right)>0$, or equivalently, $\left.\frac{d^{2}}{d \tau^{2}}\right|_{\tau=0} h\left(s_{c}+i \tau\right)=-h^{\prime \prime}\left(s_{c}\right)<0$. The real part of the function $f(\tau)=h\left(s_{c}+i \tau\right)-h\left(s_{c}\right)$ is strictly decreasing in $|\tau|$, with $\operatorname{Re} f(\tau) \leqq-$ const. $|\tau|^{1 / 2}$ as $|\tau| \rightarrow \infty$.

Proof. The analyticity of $h$ for $\operatorname{Re} s>0$ is apparent from (4.12). Critical points are solutions of

$$
h^{\prime}(s)=N^{2}-\int \frac{d^{2} k}{k^{2}+k^{4}+s}=0 .
$$

The integral is real only if $s$ is real, and is a monotone function of $s>0$ with range $(0, \infty)$. Thus, (4.16) has a unique solution $s_{c}=s_{c}\left(N^{2}\right)>0$, which obeys

$$
\begin{gathered}
\int \frac{d^{2} k}{k^{2}+k^{4}+s_{c}}=N^{2}, \\
h\left(s_{c}\right)=\int_{0}^{s_{c}} d t \int d^{2} k\left(\frac{1}{k^{2}+k^{4}+s_{c}}-\frac{1}{k^{2}+k^{4}+t}\right)<0,
\end{gathered}
$$


and

$$
h^{\prime \prime}\left(s_{c}\right)=\int \frac{d^{2} k}{\left(k^{2}+k^{4}+s_{c}\right)^{2}}>0
$$

By definition,

$$
\begin{aligned}
f(\tau) & =i \tau N^{2}-\int_{s_{c}}^{s_{c}+i \tau} d t \int \frac{d^{2} k}{k^{2}+k^{4}+t} \\
& =i \tau N^{2}-\int d^{2} k \log \left[\frac{k^{2}+k^{4}+s_{c}+i \tau}{k^{2}+k^{4}+s_{c}}\right] .
\end{aligned}
$$

Therefore

$$
\operatorname{Re} f(\tau)=-\frac{1}{2} \int d^{2} k \log \left[\frac{\left(k^{2}+k^{4}+s_{c}\right)^{2}+\tau^{2}}{\left(k^{2}+k^{4}+s_{c}\right)^{2}}\right],
$$

and hence

$$
\frac{d \operatorname{Re} f}{d \tau}=-\int d^{2} k \frac{\tau}{\left(k^{2}+k^{4}+s_{c}\right)^{2}+\tau^{2}} .
$$

This shows that $\operatorname{Re} f(\tau)$ is strictly decreasing in $|\tau|$. Scaling $k \rightarrow|\tau|^{1 / 4} k$ in (4.21), and writing $\|k\|$ for the Euclidean norm of $k$, gives

$$
\begin{aligned}
\frac{\operatorname{Re} f(\tau)}{|\tau|^{1 / 2}} & =-\frac{1}{2} \int d^{2} k \log \left[\frac{\left(\tau^{-1 / 2} k^{2}+k^{4}+\tau^{-1} s_{c}\right)^{2}+1}{\left(\tau^{-1 / 2} k^{2}+k^{4}+\tau^{-1} s_{c}\right)^{2}}\right] \\
& \leqq-\frac{1}{2} \int_{\|k\| \geqq 1} d^{2} k \log \left[1+\frac{1}{\left(\tau^{-1 / 2} k^{2}+k^{4}+\tau^{-1} s_{c}\right)^{2}}\right] \\
& \rightarrow-\frac{1}{2} \int_{\|k\| \geqq 1} d^{2} k \log \left[1+\frac{1}{k^{4}}\right], \quad \text { as }|\tau| \rightarrow \infty
\end{aligned}
$$

This proves that $\operatorname{Re} f(\tau) \leqq-$ const. $|\tau|^{1 / 2}$ as $|\tau| \rightarrow \infty$.

The next lemma provides several properties of the covariance $C_{a}^{s}(x)$.

Lemma 4.2. Let $s=\sigma+i \tau$ with $\sigma>0$ fixed.

(a) The covariance $C_{a}^{a^{-2}}(x)$ is uniformly bounded in $a, x, \tau$, and is continuous at $\tau=0$ uniformly in $a$ and $x$. Also,

$$
\lim _{a \rightarrow 0} C_{a}^{a^{-2} s_{c}}(0)=N^{2} .
$$

(b) The following identity holds:

$$
C_{a}^{s}(x)=\sum_{n \in \mathbb{Z}^{2}} \int_{\mathbb{R}^{2}} \hat{C}_{a}^{s}(k) e^{2 \pi i k \cdot(x+n)} d^{2} k .
$$

(c) Let $\tilde{\sigma}=\sigma+a^{2} m^{2}$ and $r(\tilde{\sigma}, \tau)=\max \left\{\min \left\{\tilde{\sigma}^{1 / 4}, \tilde{\sigma}^{1 / 2}\right\}, \min \left\{|\tau|^{1 / 4},|\tau|^{1 / 2}\right\}\right\}$. There is a positive constant $\delta$ (independent of $\sigma, \tau, a, m, x)$ such that

$$
\left|\int_{\mathbb{R}^{2}} \hat{C}_{a}^{a^{-2} s}(k) e^{2 \pi i k \cdot x} d^{2} k\right| \leqq K(\tilde{\sigma}) \exp \left[-a^{-1} \delta r(\tilde{\sigma}, \tau)\|x\|\right],
$$

with $K(\tilde{\sigma})=2 \int_{\mathbb{R}^{2}} d^{2} k /\left(k^{2}+k^{4}+\tilde{\sigma}\right)$. In particular, $K(\tilde{\sigma})=O(|\log \tilde{\sigma}|)$ as $\tilde{\sigma} \rightarrow 0$. 
Proof. (a) By definition,

$$
C_{a}^{a^{-2} s}(x)=\sum_{k \in \mathbb{Z}^{2}} \frac{e^{2 \pi i k \cdot x}}{a^{2} k^{4}+k^{2}+m^{2}+a^{-2} s} .
$$

Arguing as in (4.13), it is not hard to see that this is uniformly bounded and continuous as in the statement of the lemma, and, using (4.17), that (4.24) holds.

(b) The Poisson summation formula

$$
\sum_{k \in \mathbb{Z}^{2}} \frac{e^{2 \pi i k \cdot x}}{k^{2}+a^{2} k^{4}+m^{2}+s}=\sum_{n \in \mathbb{Z}^{2}} \int_{\mathbb{R}^{2}} \frac{e^{2 \pi i k \cdot(x+n)} d^{2} k}{k^{2}+a^{2} k^{4}+m^{2}+s}
$$

proves (4.25), since the left side is $C_{a}^{s}(x)$.

(c) For (4.26), we first note that scaling $k \rightarrow a^{-1} k$ gives

$$
\int_{\mathbb{R}^{2}} \hat{C}_{a}^{a^{-2} s}(k) e^{2 \pi i k \cdot x} d^{2} k=\int_{\mathbb{R}^{2}} \frac{e^{2 \pi i k \cdot x / a}}{k^{4}+k^{2}+a^{2} m^{2}+s} d^{2} k .
$$

We shall deform the $k_{1}$ - and $k_{2}$-contours into the complex plane, obtaining the desired exponential decay from the imaginary parts of the deformed contours. To simplify the notation, we define

$$
P(k)=k^{4}+k^{2}+a^{2} m^{2}+\sigma+i \tau .
$$

Then

$$
\begin{aligned}
P(k+i \kappa)= & k^{4}-2 k^{2} \kappa^{2}+\kappa^{4}-4(\kappa \cdot k)^{2}+k^{2}-\kappa^{2}+a^{2} m^{2}+\sigma \\
& +i\left(4(\kappa \cdot k) k^{2}-4(\kappa \cdot k) \kappa^{2}+2(k \cdot \kappa)+\tau\right) .
\end{aligned}
$$

We claim that there is a $\delta>0$, independent of $\tilde{\sigma}$ and $\tau$, such that

$$
|P(k+i \kappa)| \geqq \frac{1}{2} \operatorname{Re} P(k), \quad \text { if }\|\kappa\| \leqq \delta r(\tilde{\sigma}, \tau) .
$$

To prove the claim, we first note that for $A$ large (independent of $\tilde{\sigma}, \tau$ ), if $\|k\| \geqq$ $A\|\kappa\|$ then (4.31) implies

$$
|P(k+i \kappa)| \geqq \operatorname{Re} P(k+i \kappa) \geqq\left(1-O\left(A^{-2}\right)\right) \operatorname{Re} P(k) \geqq \frac{1}{2} \operatorname{Re} P(k) .
$$

For the remaining case where $\|k\| \leqq A\|\kappa\|$, it suffices to show that (4.32) holds if in addition either (i) $\|\kappa\|^{4},\|\kappa\|^{2} \leqq \delta_{1} \tilde{\sigma}$ or (ii) $\|\kappa\|^{4},\|\kappa\|^{2} \leqq \delta_{2}|\tau|$, for appropriately chosen constants $\delta_{1}$ and $\delta_{2}$. Then taking $\delta^{2}=\min \left\{1, \delta_{1}, \delta_{2}\right\}$ proves the claim (4.32). In case (ii) we can also assume that $|\tau| \geqq \tilde{\sigma}$, because otherwise the condition defining case (ii) implies that of case (i).

For case (i), we can choose $\delta_{1}>0$ (independent of $\left.\tilde{\sigma}, \tau\right)$ such that if $\|k\| \leqq$ $A\|\kappa\|$ and $\|\kappa\|^{4},\|\kappa\|^{2} \leqq \delta_{1} \tilde{\sigma}$, then (4.31) implies

$$
|P(k+i \kappa)| \geqq \operatorname{Re} P(k+i \kappa) \geqq \operatorname{Re} P(k)-O\left(\delta_{1}\right) \tilde{\sigma} \geqq \frac{1}{2} \operatorname{Re} P(k) .
$$

For case (ii), we can choose $\delta_{2}>0$ (independent of $\left.\tilde{\sigma}, \tau\right)$ such that if $\|k\| \leqq A\|\kappa\|$ and $\|\kappa\|^{4},\|\kappa\|^{2} \leqq \delta_{2}|\tau|$, then (4.31) implies

$$
|P(k+i \kappa)| \geqq|\operatorname{Im} P(k+i \kappa)| \geqq\left(1-O\left(\delta_{2}\right)\right)|\tau|+k^{4}+k^{2} \geqq \frac{1}{2} \operatorname{Re} P(k),
$$


using $k^{4}+k^{2} \leqq O\left(\delta_{2}\right)|\tau|$ in the second inequality and $|\tau| \geqq \tilde{\sigma}$ for the third. This completes the proof of (4.32).

By (4.32), the contours for $k=\left(k_{1}, k_{2}\right)$ can be displaced, without encountering any poles of the integrand, by $k \rightarrow k+i \kappa$ with $\kappa=\delta r(\tilde{\sigma}, \tau) x /\|x\|$. After absorbing a factor $2 \pi$ into $\delta$, this gives

$$
\begin{aligned}
\left|\int \frac{e^{2 \pi i k \cdot x / a} d^{2} k}{P(k)}\right| & \leqq 2 e^{-a^{-1} \delta r(\tilde{\sigma}, \tau)\|x\|} \int \frac{d^{2} k}{\operatorname{Re} P(k)} \\
& =2 e^{-a^{-1} \delta r(\tilde{\sigma}, \tau)\|x\|} \int \frac{d^{2} k}{k^{4}+k^{2}+\tilde{\sigma}} \\
& \equiv K(\tilde{\sigma}) e^{-a^{-1} \delta r(\tilde{\sigma}, \tau)\|x\|}
\end{aligned}
$$

Clearly $K(\tilde{\sigma})=O(|\log \tilde{\sigma}|)$ for small $\tilde{\sigma}>0$.

The following lemma shows that, for $s=s_{c}+i \tau$, the function $\rho(a, s)$ of (4.14) consists of a contribution depending only on $a$ and a term which is uniformly bounded.

Lemma 4.3. The function $\rho(a, s)$ can be written as $\rho(a, s)=\rho\left(a, s_{c}\right)+\gamma(a, s)$, where $\gamma\left(a, s_{c}+i \tau\right)$ is bounded uniformly in $\tau$ and small $a$, and $\lim _{\tau \rightarrow 0} \gamma\left(a, s_{c}+i \tau\right)=0$ uniformly in $a$. In addition, $\lim _{a \rightarrow 0} a^{2} \rho\left(a, s_{c}\right)=0$.

Proof. Let $s=s_{c}+i \tau$. By (4.10), (4.12) and (4.14), $\rho(a, s)=\rho\left(a, s_{c}\right)+\gamma(a, s)$ with

$$
\gamma(a, s)=-a^{-2} \int_{s_{c}}^{s} d t C_{a}^{a^{-2} t}(0)+a^{-2} \int_{s_{c}}^{s} d t \int \frac{d^{2} k}{k^{2}+k^{4}+t} .
$$

On the right side, we add and subtract the integral

$$
a^{-2} \int_{s_{c}}^{s} d t \int \frac{d^{2} k}{k^{2}+k^{4}+a^{2} m^{2}+t}
$$

and let

$$
\begin{aligned}
\gamma_{1}(a, s) & =a^{-2} \int_{s_{c}}^{s} d t \int \frac{d^{2} k}{k^{2}+k^{4}+t}-a^{-2} \int_{s_{c}}^{s} d t \int \frac{d^{2} k}{k^{2}+k^{4}+a^{2} m^{2}+t} \\
& =i m^{2} \pi \int_{0}^{\tau} d t \int_{0}^{\infty} \frac{d u}{\left(u^{2}+u+s_{c}+i t\right)\left(u^{2}+u+a^{2} m^{2}+s_{c}+i t\right)} .
\end{aligned}
$$

This is bounded uniformly in $a, \tau$ by taking absolute values under the integrals. Also, $\lim _{\tau \rightarrow 0} \gamma_{1}(a, s)=0$ uniformly in $a$.

Defining $\gamma_{2}(a, s)=\gamma(a, s)-\gamma_{1}(a, s)$ we are left with the term

$$
\gamma_{2}(a, s)=-a^{-2} \int_{s_{c}}^{s} d t C_{a}^{a^{-2} t}(0)+a^{-2} \int_{s_{c}}^{s} d t \int \frac{d^{2} k}{k^{2}+k^{4}+a^{2} m^{2}+t} .
$$


By (4.25) of Lemma 4.2,

$$
\gamma_{2}(a, s)=-i a^{-2} \int_{0}^{\tau} d t \sum_{n \neq 0} \int \hat{C}_{a}^{a^{-2}\left(s_{c}+i t\right)}(k) e^{2 \pi i k \cdot n} d^{2} k
$$

By (4.26), there are $K_{1}\left(s_{c}\right)$ and $\delta\left(s_{c}\right)>0$ such that

$$
\left|\gamma_{2}\left(a, s_{c}+i \tau\right)\right| \leqq a^{-2} \int_{0}^{\tau} d t K_{1}\left(s_{c}\right) e^{-a^{-1} \delta\left(s_{c}\right)\left(1+t^{1 / 4}\right)}
$$

This is bounded uniformly in $a$ and $\tau$, and $\lim _{\tau \rightarrow 0} \gamma_{2}(a, s)=0$ uniformly in $a$.

Finally, to prove that $\lim _{a \rightarrow 0} a^{2} \rho\left(a, s_{c}\right)=0$, we argue as above to write

$$
\begin{aligned}
a^{2} \rho\left(a, s_{c}\right)= & a^{2} m^{2} \pi \int_{0}^{s_{c}} d s \int_{0}^{\infty} \frac{d u}{\left(u^{2}+u+s\right)\left(u^{2}+u+a^{2} m^{2}+s\right)} \\
& -\int_{0}^{s_{c}} d s \sum_{n \neq 0} \int \hat{C}_{a}^{a^{-2} s}(k) e^{2 \pi i k \cdot{ }^{n} d^{2} k} .
\end{aligned}
$$

An elementary argument shows that the first term behaves like $a^{2} m^{2} \log \left(a^{2} m^{2}\right)^{-1}$. The same behaviour can be discerned for the second term, using (4.26).

4.3. Asymptotics. With the above lemmas, we are now in a position to evaluate the asymptotic behaviour of the integral $\int g(\phi) \chi_{N^{2}}\left(\|\phi\|_{2}^{2}\right) d \mu_{C_{a}}(\phi)$.

Lemma 4.4. Let $s=s_{c}+i \tau$. Suppose that $\langle g\rangle^{\left(a^{-2} s\right)}$ is polynomially bounded in $\tau$ uniformly in a, that $\lim _{a \rightarrow 0}\langle g\rangle^{\left(a^{-2} s_{c}\right)}$ exists, and that $\langle g\rangle^{\left(a^{-2} s\right)}$ is continuous at $\tau=0$ uniformly in $a$. Then

$$
\lim _{a \rightarrow 0} e^{-a^{-2} h\left(s_{c}\right)-\rho\left(a, s_{c}\right)} a^{-1} \int g(\phi) \chi_{N^{2}}\left(\|\phi\|_{2}^{2}\right) d \mu_{C_{a}}(\phi)=\frac{1}{s_{c} \sqrt{2 \pi h^{\prime \prime}\left(s_{c}\right)}} \lim _{a \rightarrow 0}\langle g\rangle^{\left(a^{-2} s_{c}\right)} .
$$

Proof. The integral on the left side of (4.44) is given by (4.15), in which we take $\sigma=s_{c}$. The assumption that $\langle g\rangle^{\left(a^{-2} s\right)}$ is polynomially bounded in $\tau$, together with Lemma 4.3 and the bound on $f$ of Lemma 4.1, allow us to apply the dominated convergence theorem to take the limit $A \rightarrow \infty$ in (4.15), yielding

$$
\int g(\phi) \chi_{N^{2}}\left(\|\phi\|_{2}^{2}\right) d \mu_{C_{a}}(\phi)=\frac{1}{2 \pi i} \int_{\Gamma_{s_{c}}} \frac{d s}{s} e^{a^{-2} h(s)+\rho(a, s)}\langle g\rangle^{\left(a^{-2} s\right)} .
$$

Thus (4.44) is equivalent to

$$
\lim _{a \rightarrow 0} a^{-1} \int_{-\infty}^{\infty} \frac{d \tau}{s_{c}+i \tau} e^{a^{-2} f(\tau)} e^{\gamma\left(a, s_{c}+i \tau\right)}\langle g\rangle^{\left(a^{-2}\left(s_{c}+i \tau\right)\right)}=\frac{\sqrt{2 \pi}}{s_{c} \sqrt{h^{\prime \prime}\left(s_{c}\right)}} \lim _{a \rightarrow 0}\langle g\rangle^{\left(a^{-2} s_{c}\right)},
$$

or writing

$$
p(a, \tau)=\frac{1}{s_{c}+i \tau} e^{\gamma\left(a, s_{c}+i \tau\right)}\langle g\rangle^{\left(a^{-2}\left(s_{c}+i \tau\right)\right)} \quad \text { and } \quad p(0,0)=\lim _{a \rightarrow 0} p(a, 0),
$$


to

$$
\lim _{a \rightarrow 0} a^{-1} \int_{-\infty}^{\infty} e^{a^{-2} f(\tau)} p(a, \tau) d \tau=\frac{\sqrt{2 \pi}}{\sqrt{h^{\prime \prime}\left(s_{c}\right)}} p(0,0)
$$

By Lemma 4.3, $p$ satisfies the same hypotheses as $\langle g\rangle^{\left(a^{-2} s\right)}$.

By Taylor's theorem and Lemma 4.1,

$$
f(\tau)=-\frac{1}{2} h^{\prime \prime}\left(s_{c}\right) \tau^{2}+r(\tau)
$$

where for $\tau$ in a sufficiently small interval $I=[-\varepsilon, \varepsilon]$,

$$
|r(\tau)| \leqq c_{1}|\tau|^{3} \quad \text { and } \quad-\frac{1}{2} h^{\prime \prime}\left(s_{c}\right) \tau^{2}+c_{1}|\tau|^{3} \leqq-\frac{1}{4} h^{\prime \prime}\left(s_{c}\right) \tau^{2} .
$$

By Lemma 4.1 and our control on $p$,

$$
\left|a^{-1} \int_{I^{c}} e^{a^{-2} f(\tau)} p(a, \tau) d \tau\right| \leqq a^{-1} \int_{I^{c}} e^{a^{-2} \operatorname{Re} f(\tau)}|p(a, \tau)| d \tau \leqq C e^{-c(\varepsilon) a^{-2}}
$$

and we are reduced to the contribution from $I$.

By (4.49),

$$
\begin{aligned}
& a^{-1} \int_{I} e^{a^{-2} f(\tau)} p(a, \tau) d \tau=a^{-1} p(a, 0) \int_{I} e^{-a^{-2} h^{\prime \prime}\left(s_{c}\right) \tau^{2} / 2} d \tau \\
& +a^{-1} \int_{I} e^{-a^{-2} h^{\prime \prime}\left(s_{c}\right) \tau^{2} / 2}\left[e^{a^{-2} r(\tau)} p(a, \tau)-p(a, 0)\right] d \tau
\end{aligned}
$$

The first term in (4.52) is

$$
a^{-1} p(a, 0) \int_{I} e^{-a^{-2} h^{\prime \prime}\left(s_{c}\right) \tau^{2} / 2} d \tau=p(a, 0) \int_{a^{-1} I} e^{-h^{\prime \prime}\left(s_{c}\right) \tau^{2} / 2} d \tau
$$

which converges to the right side of (4.48). It remains to show that the second term in (4.52) goes to zero as $a \rightarrow 0$. For this we use

$$
\begin{aligned}
\left|e^{a^{-2} r(\tau)} p(a, \tau)-p(a, 0)\right| \leqq & e^{a^{-2}|r(\tau)|}|p(a, \tau)-p(a, 0)| \\
& +e^{a^{-2}|r(\tau)|}\left|a^{-2} r(\tau) p(a, 0)\right|
\end{aligned}
$$

By (4.50), the contribution to the second term in (4.52) due to the first term on the right side of (4.54) is at most

$$
a^{-1} \int_{I} e^{-a^{-2} h^{\prime \prime}\left(s_{c}\right) \tau^{2} / 4}|p(a, \tau)-p(a, 0)| d \tau .
$$

This can be made as small as desired by taking $\varepsilon$ sufficiently small, by the fact that $p(a, \tau)$ is continuous at $\tau=0$ uniformly in $a$. By $(4.50)$, the contribution to the second term in (4.52) due to the second term on the right side of (4.54) is at most

$a^{-1}|p(a, 0)| \int_{I} e^{-a^{-2} h^{\prime \prime}\left(s_{c}\right) \tau^{2} / 4} c_{1} a^{-2}|\tau|^{3} d \tau \leqq O(a)|p(a, 0)|=O(a)$. 
Proof of Lemma 1.3. (a) By Lemma 4.4 with $g=1$,

$$
S_{a} \sim \frac{a}{s_{c} \sqrt{2 \pi h^{\prime \prime}\left(s_{c}\right)}} e^{a^{-2}\left[h\left(s_{c}\right)+a^{2} \rho\left(a, s_{c}\right)\right]} .
$$

The desired result then follows from Lemmas 4.1 and 4.3, with $\alpha\left(N^{2}\right)=-h\left(s_{c}\left(N^{2}\right)\right)$.

(b) Let $g(\phi)=|\phi(0)|^{2} e^{\lambda N^{2}|\phi(0)|^{2}}$. The desired result will follow from Lemma 4.4, once we show that if $\lambda<N^{-4}$, then $\langle g\rangle^{\left(a^{-2} s\right)}$ obeys the hypotheses of Lemma 4.4 and $\lim _{a \rightarrow 0}\langle g\rangle^{\left(a^{-2} s_{c}\right)}=N^{2} /\left(1-\lambda N^{4}\right)^{2}$.

The Gaussian expectation $\langle g\rangle^{\left(a^{-2} s\right)}$ is analytic in a half plane containing the contour $s=s_{c}+i \tau$, provided the numerator $\int \exp \left(-a^{-2} s\|\phi\|_{2}^{2}\right) g(\phi) d \mu_{C_{a}}$ is absolutely convergent for some real $s<s_{c}$, since the denominator $e^{Q\left(a^{-2} s\right)} \neq 0$ is analytic. For $s$ real and near $s_{c}, \phi(0)$ is a complex Gaussian random variable with distribution

$$
Z^{-1} \exp \left[-C_{a}^{a^{-2} s}(0)^{-1}|\phi(0)|^{2}\right] d \phi(0) .
$$

Therefore $\langle g\rangle^{\left(a^{-2} s\right)}$ is analytic in a half plane containing the contour when $\lambda N^{2}<$ $C_{a}^{a^{-2} s_{c}}(0)^{-1}$, which by (4.24) is equivalent to $\lambda<N^{-4}$. For such values of $\lambda$, and $s$ real and near $s_{c}$,

$$
\begin{aligned}
\langle g\rangle^{\left(a^{-2} s\right)} & =\frac{\partial}{\partial\left(\lambda N^{2}\right)} Z^{-1} \int e^{-C_{a}^{a^{-2}}(0)^{-1}|\phi(0)|^{2}} e^{\lambda N^{2}|\phi(0)|^{2}} d \phi(0) \\
& =\frac{\partial}{\partial\left(\lambda N^{2}\right)} \frac{1}{1-\lambda N^{2} C_{a}^{a^{-2} s}(0)} \\
& =\frac{C_{a}^{a^{-2} s}(0)}{\left(1-\lambda N^{2} C_{a}^{a^{-2} s}(0)\right)^{2}} .
\end{aligned}
$$

By analytic continuation, this also holds when $s$ lies on the contour.

By Lemma 4.2(a), the continuity hypothesis of Lemma 4.4 holds. The hypothesis that $\langle g\rangle^{\left(a^{-2} s\right)}$ is polynomially bounded in $\tau$ follows from the uniform bound of Lemma 4.2(a), together with the fact that by definition the real part of $C_{a}^{a^{-2} s}(0)$ is bounded above by $C_{a}^{a^{-2} s_{c}}(0)$, so by (4.24) the denominator of (4.59) is bounded away from zero. Existence of the limit required as a hypothesis of Lemma 4.4 follows from (4.24). Thus Lemma 4.4 gives

$$
\lim _{a \rightarrow 0}\left\langle|\phi(0)|^{2} e^{\lambda N^{2}|\phi(0)|^{2}}\right\rangle_{a}=\frac{N^{2}}{\left(1-\lambda N^{4}\right)^{2}},
$$

which is (1.23).

(c) We apply Lemma 4.4 with $g=F$ of the form (1.13). It suffices to show that $\langle F\rangle^{\left(a^{-2} s\right)}$ obeys the hypotheses of Lemma 4.4 and that $\lim _{a \rightarrow 0}\langle F\rangle^{\left(a^{-2} s\right)}=\langle F\rangle_{0}$.

The Gaussian expectation $\langle F\rangle^{\left(a^{-2} s\right)}$ is a correlation function of the Gaussian measure with covariance $C_{a}^{a^{-2}}(x)$. By Wick's theorem [10], $\langle F\rangle^{\left(a^{-2} s\right)}$ is given by a sum of Feynman diagrams having propagator $C_{a}^{a^{-2} s}(x)$. If $m_{j}=n_{j}$ for all $j$, there is a contribution

$$
\prod_{j=1}^{n} m_{j} ! C_{a}^{a^{-2} s}(0)^{m_{j}}
$$


arising from the diagrams having only self-lines, together possibly with other diagrams containing at least one propagator that is not a self-line. The latter type of diagram is the only type that occurs when $m_{j} \neq n_{j}$ for some $j$. Such diagrams are defined as multiple integrals over the torus of products of factors $C_{a}^{a^{-2}}(x-y)$. By Lemma 4.2(a), the hypotheses of Lemma 4.4 are all satisfied and thus $\lim _{a \rightarrow 0}\langle F\rangle_{a}$ is equal to the limiting value of the sum of the Feynman diagrams, with $s=s_{c}$, as $a \rightarrow 0$.

By (4.24), with $s=s_{c}$ the limit of (4.61) is the desired limit $\prod_{j=1}^{n} m_{j} ! N^{2 m_{j}}=$ $\langle F\rangle_{0}$. By Lemma 4.2,

$$
\lim _{a \rightarrow 0} C_{a}^{a^{-2} s_{c}}(x)=0, \quad(x \neq 0)
$$

sufficiently rapidly that any Feynman diagram with a line that is not a self-line goes to zero in the limit.

We end with a heuristic remark pertaining to the discussion on the effect of scaling the temperature to the critical point in the spherical model, in Sect. 1.3.2.

Remark 4.5. Scaling the temperature to the critical point in the spherical model corresponds to introducing a factor $\beta(a)^{-1}$, with $\lim _{a \rightarrow 0} \beta(a)=\infty$, into the covariance. Scaling the field as $\phi \rightarrow \beta(a)^{-1 / 2} \phi$, this insertion of $\beta(a)$ is the same as instead replacing $N^{2}$ by $N^{2} \beta(a)$ in the constraint. As in (4.17), in this case the critical value $s_{c}=s_{c}(a)$ is determined by

$$
N^{2} \beta(a)=\int_{\mathbb{R}^{2}} \frac{d^{2} k}{k^{2}+k^{4}+s_{c}(a)} .
$$

For $\beta(a) \rightarrow \infty$, we must take $s_{c}(a) \rightarrow 0$, so that roughly $N^{2} \beta(a) \approx\left|\log s_{c}(a)\right|$. As in the proof of Lemma 1.3(c), the two-point function in the limit $a \rightarrow 0$ is asymptotic to

$$
C_{a}^{a^{-2} s_{c}(a)}(x)=\sum_{k} \frac{e^{2 \pi i k \cdot x}}{k^{2}+a^{2} k^{4}+a^{-2} s_{c}(a)} .
$$

This is nontrivial if $s_{c}$ is proportional to $a^{2}$ and hence $N^{2} \beta(a) \approx \log a^{-2}$, which corresponds to Wick ordering the constraint. In this case the limiting two-point function is of the form $\sum_{k} e^{2 \pi i k \cdot x} /\left(k^{2}+1\right)$, which is the two-point function of a massive Gaussian field on the torus.

Acknowledgements. We are grateful to Jean Bricmont for suggesting the connection between the invariant measure construction and the Berlin-Kac spherical model. The work of D.C.B. was supported in part by NSF grant DMS 9401028. The work of G.S. was supported in part by NSERC grant A9351.

\section{References}

1. Aragão de Carvalho, C., Caracciolo, S., Fröhlich, J.: Polymers and $g|\phi|^{4}$ theory in four dimensions. Nucl. Phys. B 215 [FS7], 209-248 (1983)

2. Baxter, R.J.: Exactly Solved Models in Statistical Mechanics. London: Academic Press, 1982

3. Berlin, T.H., Kac, M.: The spherical model of a ferromagnet. Phys. Rev. 86, 821-835 (1952)

4. Bidégaray, B.: Invariant measures for some partial differential equations. Physica D 82, 340-364 (1995) 
5. Bolthausen, E., Schmock, U.: On self-attracting $d$-dimensional random walks. To appear in Ann. Probab.

6. Bourgain, J.: Periodic nonlinear Schrödinger equation and invariant measures. Commun. Math. Phys. 166, 1-26 (1994)

7. Bourgain, J.: Time evolution in Gibbs measures for the nonlinear Schrödinger equations. In: D. Iagolnitzer, ed., Proceedings of XIth International Congress of Mathematical Physics, Cambridge, MA: International Press, 1995, pp. 543-547

8. Bourgain, J.: Invariant measures for the $2 d$-defocusing nonlinear Schrödinger equation. Commun. Math. Phys. 176, 421-445 (1996)

9. Brydges, D.C., Slade, G.: The diffusive phase of a model of self-interacting walks. Probab. Th. Rel. Fields 103, 285-315 (1995)

10. Glimm, J., Jaffe, A.: Quantum Physics, A Functional Integral Point of View. Berlin: Springer, 2nd edition, 1987

11. Jaffe, A.: Ann Arbor lecture. May, 1994

12. Le Gall, J.-F.: Exponential moments for the renormalized self-intersection local time of planar Brownian motion. In: J. Azéma, P.A. Meyer and M. Yor, eds., Séminaire de Probabilités XXVIII. Lecture Notes in Mathematics \#1583, Berlin: Springer, 1994

13. Lebowitz, J.L., Rose, H.A., Speer, E.R.: Statistical mechanics of the nonlinear Schrödinger equation. J. Stat. Phys. 50, 657-687 (1988)

14. Lebowitz, J.L., Rose, H.A., Speer, E.R.: Statistical mechanics of the nonlinear Schrödinger equation. II. Mean field approximation. J. Stat. Phys. 54, 17-56 (1989)

15. McKean, H.P.: Statistical mechanics of nonlinear wave equations (4): Cubic Schrödinger. Commun. Math. Phys. 168, 479-491 (1995). Erratum: Commun. Math. Phys. 173, 675 (1995)

16. Simon, B.: The $P(\phi)_{2}$ Euclidean (Quantum) Field Theory. Princeton, NJ: Princeton University Press, 1974

17. Strauss, W.A.: Existence of solitary waves in higher dimensions. Commun. Math. Phys. 55, 149-162 (1977)

18. Strauss, W.A.: Nonlinear Wave Equations. Providence, RI: American Mathematical Society, 1989

19. Weinstein, M.I.: Nonlinear Schrödinger equations and sharp interpolation estimates. Commun. Math. Phys. 87, 567-576 (1983)

20. Zhidkov, P.E.: On an invariant measure for a nonlinear Schrödinger equation. Soviet Math. Dokl. 43, 431-434 (1991)

21. Zhidkov, P.E.: On invariant measures for some infinite-dimensional dynamical systems. Ann. Inst. H. Poincaré - Phys. Théor. 62, 267-287 (1995)

Communicated by A. Jaffe 